\title{
Approximation operators by using finite family of reflexive relations
}

\author{
H. M. Abu-Donia ${ }^{1,3 *}$, A. S. Salama ${ }^{2,3}$ \\ ${ }^{1}$ Department of Mathematics, Faculty of Science, Zagazig University, Zagazig, Egypt \\ 2 Department of Mathematics, Faculty of Science, Tanta University, , Tanta Egypt \\ ${ }^{3}$ Department of Mathematics, Faculty of Science, Shaqra University, El-Dawadmi, K. S. A. \\ *Corresponding author E-mail: donia_1000@yahoo.com
}

Copyright (C)2015 H. M. Abu-Donia, A. S. Salama. This is an open access article distributed under the Creative Commons Attribution License, which permits unrestricted use, distribution, and reproduction in any medium, provided the original work is properly cited.

\begin{abstract}
In this paper, we generalize the two types of Yao's lower and upper approximations, using finite number of reflexive relations. Moreover, we give a comparison between these types and study some properties.
\end{abstract}

Keywords: Rough set, Lower approximations, Upper approximations, Right neighborhood, Reflexive relation, Accuracy measure.

\section{Introduction}

The rough set theory has been introduced by Z. Pawlak as a mathematical approach to deal with vagueness and uncertainty in data analysis $[23,27]$. The rough set theory $[25,27,57]$ is based on the assumption that some information about elements of the universe is available. Two objects can be indiscernible concerning available information as with some objects the same information can be associated. Thus, information associated with objects of the universe induced an indiscernibility relation in this universe. This indiscernibility relation can be employed in order to define approximations of sets or relations and therefore, the concept of the rough set is introduced. This set is characterized by a pair of precise concepts called the lower and upper approximations. The lower and upper approximation operators are related to the necessity (box) and possibility (diamond) operators of modal logic [5], and the interior and closure operators in topological space $[9,12,16,17,19,38,39,44]$. Zakowski [47] studied a set of axioms on approximation operators, and Comer [10] investigated axioms on approximation operators in relation to cylindric algebras within the context of Pawlak information systems [28]. Mordeson [20] investigated the axiomatic characterization of approximation operators defined by Covers, and Thiele [38] explored the axiomatic characterization within modal logic. The most important axiomatic studies for crisp rough sets were done by Yao et al. [43, 44, 45, 46], In the theory of generalized rough sets, the properties and applications of various models have been extensively discussed. In fact, a systematic study of definable concepts in various generalized rough set models would provide better understanding to these models, and reasonable suggestions to construct new models for generalized rough sets, and could be beneficial to both theoretical and practical studies of rough set theory. It is meaningful to note that Cattaneo [7] extended Pawlak's model to a class of abstract models based on bounded posets (poset based model for short). In his work, Cattaneo emphasized the role of definable elements (here, elements are used to represent concepts) in constructing rough set models. Also, Cattaneo [8] used a special class of relations to obtain two kinds of approximations. Wei and Wen [39] defined a measure of roughness based on generalized rough 
sets with the new approximations and proved some properties of the measure. Pawlak and Skowron [22] presented approximation spaces based on the neighborhood of objects and on inclusion measures of the neighborhood into concepts and defined the inductive extensions of approximation spaces. Pawlak [25] defined the rough probability using equivalence relations; that is, he associated each event with an interval whose end points are lower and upper probabilities. E. A. Rady et. al. [32] introduced a general approach for computing lower and upper probabilities using a general relation instead of the equivalence relation in Pawlak's approach. The classical rough set theory is based on equivalence relations, but it has been extended to relation based rough sets [34, 41, 42, 43, 56], covering based rough sets $[18,49,51,52,53,54,55]$ and fuzzy rough sets $[13,30,41,42]$. In $[5,38,39,40]$, an extensive research on the binary relation based rough sets was done. These authors started from the properties of binary relations, for example, reflexivity, symmetry, and transitivity, to investigate the essential properties of the lower and upper approximation operations generated by such relations. Many researchers have generalized the notion of approximation operators by using non-equivalence relations, see, e.g., [21, 29, 35, 42, 44, 46, 48, 50]. It is possible to obtain the upper and lower bounds by eliminating the transitivity, reflexivity, and symmetry axioms. In [47], for example, Yao studied the general properties of rough sets which resulted from these axioms. Many authors discussed approximations with more than one lower-upper approximation pair [14, 31, 33]. In [4] Abu-Donia discussed three types of lower and upper approximations of any set with respect to any relation based on right neighborhood and generalized these three types of approximations into two ways by using a finite number of any binary relations, also some types of approximation of rough sets are discussed, see, e. g. [1, 2, 3]. The notion of approximation operators can also be generalized by using a covering of the universe [21, 29]. Yao [45] introduced and investigated the notion of generalized approximation space for any binary reflexive relation $\mathrm{R}$ by using the right neighborhood concepts and compared between the generalized approximations and Pawlak's approximations for reflexive relation. In this paper, we use a finite number of binary relations to introduce two types of lower and upper approximations which are generalizations for approximation defined by Yao [42]. Finally, we illustrate an example to show the importance of our approximations.

\section{Preliminary notes}

Let $U$ be a non-empty set called universe, and $E$ be an equivalence relation on $U$. The pair $(U, E)$ is called an approximation space. Let $[x]_{E}$ denote the equivalence class for an element $x \in U$. Let $A$ be subset of $U$. A rough set corresponding to $A$ is the ordered pair $(\underline{E}(A), \bar{E}(A))$, where $\underline{E}(A)$ and $\bar{E}(A)$ are defined as follows:

$$
\begin{gathered}
\underline{E}(A)=\left\{x \in U:[x]_{E} \subseteq A\right\} \quad \text { (called lower approximation of A). } \\
\bar{E}(A)=\left\{x \in U:[x]_{E} \cap A \neq \emptyset\right\} \quad \text { (called upper approximation of A). }
\end{gathered}
$$

Obviously, we have $\underline{E}(A) \subseteq A \subseteq \bar{E}(A)$. The lower approximation of $A$ contains the elements $x$ so that all the elements that are indistinguishable from $x$ are in $A$. The upper approximation of $A$ contains the elements $x$ so that at least one element which is indistinguishable from $x$ belongs to $A$.

The lower and upper approximations are used to divide the universe into three regions with respect to any subset $A \subseteq U:$

$B \bar{N} D(A)=\bar{E}(A)-\underline{E}(A)$.

$\operatorname{POS}(A)=\underline{E}(A)$.

$N E G(A)=\bar{U}-\bar{E}(A)$.

An element of the negative region $N E G(A)$ definitely doesn't belong to $A$, an element of the positive region $\operatorname{POS}(A)$ definitely belongs to $A$, and an element of the boundary region $B N D(A)$ only possibly belongs to $A$.

The generalized model of rough sets called Variable Precision Rough Set model (VPRS-model). The VPRSmodel, proposed by Ziarko [58] inherits all basic properties of the original rough set model and aims at handling uncertain information.

The fundamental notion introduced by the VPRS-model is the generalization of the standard inclusion relation called majority inclusion relation.

The following are definitions according to Ziarko [58]: 
Definition 2.1 Let $A$ and $B$ be non-empty subsets of a finite universe $U$. Then the measure of the relative degree of misclassification of set $A$ with respect to set $B$ denoted by $C(A, B)$ is defined as:

$$
C(A, B)= \begin{cases}1-\frac{|A \cap B|}{|A|}, & \text { if }|A|>0 \\ 0, & \text { if }|A|=0\end{cases}
$$

where "|. |" denotes the cardinality of the set.

Definition 2.2 The inclusion relationship between $A$ and $B$ without explicitly using a general quantifier:

$A \subseteq B$ if $C(A, B)=0$

Notice that: The majority requirement implies that more than $50 \%$ of $A$ elements should be in common with $B$. According to the majority requirement, the admissible classification error $\beta$ must be within the range of $0 \leq \beta<0.5$.

Definition 2.3 $A$ is included in $B$ with respect to $\beta(A \subseteq B$ 을 $C(A, B) \leq \beta$.

One of the important results in the rough set theory is the deviation between the inclusion in the rough set theory and the ordinary set theory. There is one type of inclusion in the ordinary set theory $(A \subseteq B)$ but in the rough set theory we have many types of inclusion of sets, as shown in the following definition.

Definition 2.4 [25] Let $(U, R)$ be an approximation space. For any $A, B \subseteq U$, then we say:

(i) $A$ is roughly bottom included in $B(A \subset \mathcal{\sim} B)$ if $\underline{E}(A) \subset \underline{E}(B)$.

(ii) $A$ is roughly top included in $B(A \stackrel{\sim}{\subset} B)$ if $\bar{E}(A) \subset \bar{E}(B)$.

(iii) $A$ is roughly included in $B(A \underset{\sim}{\sim} B)$ if $(A \underset{\sim}{\sim} B)$ and $(A \stackrel{\sim}{\sim} B)$

From the above we see that the inclusion of the neighborhood in concepts was applied via many cases such as

(1) The classical inclusion.

(2) The VPRS-inclusion by Ziarko [58]

(3) The rough (resp. top and bottom) inclusion by Pawlak [25]

In our case, the inclusion used in the concept is the classical inclusion.

\section{Yao's rough set approximations}

In this section we discuss three kinds of rough set approximations based on the right neighborhood.

Definition 3.1 For the pair $(U, R)$, where $U$ is a finite universe, $U \neq \emptyset$ and $R$ being any binary relation, let $x R$ be the right neighborhood defined as: $x R=\{y \in U: x R y\}$.

Definition 3.2 Let $R$ be any binary relation on a nonempty set $U$. For any the set $A \subseteq U$, the lower and upper approximations of $A$ according to $R$ are then defined as:

$$
\begin{gathered}
\underline{R}(A)=\{x \in U: x R \subseteq A\}, \\
\bar{R}(A)=\{x \in U: x R \cap A \neq \emptyset\} .
\end{gathered}
$$

Obviously, if $R$ is an equivalence relation, then $x R=[x]_{R}$ and these definitions are equivalent to the original Pawlak's definitions.

where $[x]_{R}$ is called an equivalence class of $x \in U$.

We list the properties that are of interest in the theory of rough sets, let $A, B \subseteq U$ :

$\left(L_{1}\right) \underline{R}(A)=\left(\bar{R}\left(A^{c}\right)\right)^{c}$, where $A^{c}$ denotes the complement of $A$ in $U$.

$\left(L_{2}\right) \underline{R}(U)=U$. 
$\left(L_{3}\right) \underline{R}(A \cap B)=\underline{R}(A) \cap \underline{R}(B)$.

$\left(L_{4}\right) \underline{R}(A \cup B) \supseteq \underline{R}(A) \cup \underline{R}(B)$.

$\left(L_{5}\right) A \subseteq B \Rightarrow \underline{R}(A) \subseteq \underline{R}(B)$.

$\left(L_{6}\right) \underline{R}(\emptyset)=\emptyset$.

$\left(L_{7}\right) \underline{R}(A) \subseteq A$.

$\left(L_{8}\right) A \subseteq \underline{R}(\bar{R}(A))$.

$\left(L_{9}\right) \underline{R}(A)=\underline{R}(\underline{R}(A))$.

$\left(L_{10}\right) \bar{R}(A)=\underline{R}(\bar{R}(A))$.

$\left(U_{1}\right) \bar{R}(A)=\left(\underline{R}\left(A^{c}\right)\right)^{c}$.

$\left(U_{2}\right) \bar{R}(\emptyset)=\emptyset$.

$\left(U_{3}\right) \bar{R}(A \cup B)=\bar{R}(A) \cup \bar{R}(B)$.

$\left(U_{4}\right) \bar{R}(A \cap B) \subseteq \bar{R}(A) \cap \bar{R}(B)$.

$\left(U_{5}\right) A \subseteq B \Rightarrow \bar{R}(A) \subseteq \bar{R}(B)$.

$\left(U_{6}\right) \bar{R}(U)=U$.

$\left(U_{7}\right) A \subseteq \bar{R}(A)$.

$\left(U_{8}\right) A \supseteq \bar{R}(\underline{R}(A))$.

$\left(U_{9}\right) \bar{R}(A)=\bar{R}(\bar{R}(A))$.

$\left(U_{10}\right) \underline{R}(A)=\bar{R}(\underline{R}(A))$.

(CO) $\underline{R}\left(A^{c} \cup B\right) \subseteq(\underline{R}(A))^{c} \cup \underline{R}(B)$.

(LU) $\underline{R}(A) \subseteq \bar{R}(A)$.

By using Definition 3.2, we can define the accuracy measure of a set $A[41]$ as

$$
\alpha(A)=\frac{|\underline{R}(A)|}{|\bar{R}(A)|} .
$$

Definition 3.3 [42] Let $R$ be any binary relation on a nonempty set $U$ and $x R$ be the right neighborhood of $x$ according to $R$ for any set $A \subseteq U$. The definition for lower and upper approximations of $A$ according to $R$ is defined as:

$$
\begin{gathered}
\underline{R^{\prime}}(A)=\bigcup\{x R: x R \subseteq A\}, \\
\bar{R}^{\prime}(A)=\left(\underline{R^{\prime}}\left(A^{c}\right)\right)^{c} .
\end{gathered}
$$

So, the accuracy measure of a set $A$ will be defined as:

$$
\alpha^{\prime}(A)=\frac{\left|\underline{R}^{\prime}(A)\right|}{\left|\bar{R}^{\prime}(A)\right|} .
$$


Table 1: Upper and lower approximations under reflexive relation by using Definition 3.3 and Definition 3.4

\begin{tabular}{ccc}
\hline Properties & Definition 3.3 & Definition 3.4 \\
\hline$L_{1}$ & $\star$ & $\star$ \\
$L_{2}$ & $\star$ & $\star$ \\
$L_{3}$ & & $\star$ \\
$L_{4}$ & $\star$ & $\star$ \\
$L_{5}$ & $\star$ & $\star$ \\
$L_{6}$ & $\star$ & \\
$L_{7}$ & $\star$ & \\
$L_{8}$ & & $\star$ \\
$L_{9}$ & $\star$ & $\star$ \\
$L_{10}$ & & $\star$ \\
$U_{1}$ & $\star$ & $\star$ \\
$U_{2}$ & $\star$ & $\star$ \\
$U_{3}$ & & $\star$ \\
$U_{4}$ & $\star$ & \\
$U_{5}$ & $\star$ & \\
$U_{6}$ & $\star$ & \\
$U_{7}$ & $\star$ & \\
$U_{8}$ & & \\
$U_{9}$ & $\star$ & \\
$U_{10}$ & & \\
$C O$ & & \\
$L U$ & $\star$ & \\
\hline
\end{tabular}

Definition 3.4 [42] Let $R$ be any binary relation on a non-empty set $U$ and $x R$ be the right neighborhood of $x$ according to $R$, for any set $A \subset U$. The definition for lower and upper approximations of $A$ according to $R$ is defined as:

$$
\begin{gathered}
\underline{R}^{\prime \prime}(A)=\left(\bar{R}^{\prime \prime}\left(A^{c}\right)\right)^{c}, \\
\bar{R}^{\prime \prime}(A)=\bigcup\{x R: x R \cap A \neq \emptyset\} .
\end{gathered}
$$

So, the accuracy measure of a set $A$ will be defined as:

$$
\alpha^{\prime \prime}(A)=\frac{\left|\underline{R}^{\prime \prime}(A)\right|}{\left|\bar{R}^{\prime \prime}(A)\right|} .
$$

For any reflexive relation $R$ on a non-empty set $U$, the comparison between the properties of rough sets depending on Definition 3.3 and Definition 3.4 are shown in Table 1.

$\star$ indicates that the property is satisfied This table shows the comparison between the properties of rough sets depending on Definition 3.3 and Definition 3.4.

Remark 3.5 If a binary relation $R$ on a non-empty set $U$ is not reflexive, not transitive and not symmetric, then the following properties are not satisfied:

(1) $\underline{R}(\phi)=\phi$

(2) $\bar{R}(U)=U$

(3) $\underline{R}(A) \subseteq A$

(4) $A \subseteq \bar{R}(A)$

The following example shows Remark 3.5. 
Example 3.6 Let $U=\{a, b, c, d\}$ and consider $R=\{(a, a),(a, d),(c, c),(d, b)\}$. Consequently, $a R=\{a, d\}, b R=\phi$, $c R=\{c\}$ and $d R=\{b\}$. If $A=\{b\}$, then we have:

(1) $\underline{R}(\phi)=\{x \in U: x R \subseteq \phi\}=\{b\} \neq \phi$

(2) $\bar{R}(U)=\{x \in U: x R \cap U \neq \phi\}=\{a, b, c\} \neq U$

(3) $\underline{R}(A)=\{x \in U: x R \subseteq A\}=\{b, d\} \nsubseteq A$

(4) $\bar{R}(A)=\{x \in U: x R \cap A \neq \phi\}=\{d\} \nsupseteq A$

Although this method has opened the way for the use of general relation, the essential properties of the lower and upper approximation are not satisfied (see Example 3.6). Consequently, Yao pointed out that it required additional properties on the binary relation to satisfy the basic properties for lower and upper approximation.

\section{New Types of Generalization}

In this section, we introduce two types of lower and upper approximations by using finite number of binary relations. These types of approximations are generalizations for Definition 3.3 and Definition 3.4.

Definition 4.1 Let $\left\{R_{i}: i=1,2, \ldots, n\right\}$ be a family of binary relations on a non-empty set $U$, for any set $A \subseteq U$. We can introduce a definition for n-lower and n-upper approximations of $A$ according to $R_{i}$ as following:

$$
\underline{\operatorname{apr}}^{\diamond}(A)=\bigcup_{i=1}^{n} \underline{R}_{i}^{\prime}(A) \quad \text { and } \quad \overline{\operatorname{apr}}^{\diamond}(A)=\left(\underline{\operatorname{apr}}^{\diamond}\left(A^{c}\right)\right)^{c},
$$

where $\underline{R}_{i}^{\prime}(A)$ is the lower approximation defined in Definition 3.3 .

Remark 4.2 Definition 3.1. is a generalized form of Definition of Khan et al. [11].

We can define the accuracy measure of $A$ according to reflexive relations $R_{i}$, as the following:

$$
\alpha^{\diamond}(A)=\frac{\left|\underline{a p r} r^{\diamond}(A)\right|}{\left|\overline{a p r} r^{\diamond}(A)\right|} .
$$

Proposition 4.3 For a finite family of reflexive relations $\left\{R_{i}: i=1,2, \ldots, n\right\}$ on a non-empty set $U$, the following properties are hold for every $A, B \subseteq U$ :

$$
\begin{aligned}
& \left(L_{2}\right) \operatorname{apr}^{\diamond}(U)=U \text {. } \\
& \left(L_{4}\right) \underline{a p r}^{\diamond}(A \cup B) \supseteq \underline{a p r}^{\diamond}(A) \cup \underline{a p r}{ }^{\diamond}(B) . \\
& \left(L_{5}\right) A \subseteq B \Longrightarrow \underline{a p r} \underline{v}^{\diamond}(A) \subseteq \underline{a p r}{ }^{\diamond}(B) . \\
& \left(L_{6}\right) \underline{a p r}^{\diamond}(\emptyset)=\emptyset . \\
& \left(L_{7}\right) \operatorname{apr}^{\diamond}(A) \subseteq A . \\
& \left(L_{9}\right) \underline{a p r}^{\diamond}\left(\underline{\operatorname{apr}}{ }^{\diamond}(A)\right)=\underline{a p r}{ }^{\diamond}(A) \\
& \left(U_{2}\right) \overline{a p r}^{\diamond}(\emptyset)=\emptyset \text {. } \\
& \left(U_{4}\right) \overline{a p r}^{\diamond}(A \cap B) \subseteq \overline{a p r}^{\diamond}(A) \cap \overline{a p r}^{\diamond}(B) . \\
& \left(U_{5}\right) A \subseteq B \Longrightarrow \overline{a p r}^{\diamond}(A) \subseteq \overline{\operatorname{apr}}^{\diamond}(B) . \\
& \left(U_{6}\right) \overline{a p r}^{\diamond}(U)=U \text {. } \\
& \left(U_{7}\right) A \subseteq \overline{a p r}^{\diamond}(A) \text {. }
\end{aligned}
$$




$$
\begin{aligned}
& \left(U_{9}\right) \overline{a p r}^{\diamond}\left(\overline{a p r}^{\diamond}(A)\right)=\overline{a p r}^{\diamond}(A) \\
& (L U) \underline{a p r}^{\diamond}(A) \subseteq \overline{a p r}^{\diamond}(A) .
\end{aligned}
$$

Proof. $\left(L_{2}\right)$. As $\underline{\operatorname{apr}}^{\diamond}(U)=\bigcup_{i=1}^{n} \underline{R}_{i}^{\prime}(U)$, but $\underline{R}_{i}^{\prime}(U)=U \forall i=1,2,3, \ldots, n$. So, $\underline{\operatorname{apr}}^{\diamond}(U)=U$.

$\left(L_{4}\right)$. As $x \in \underline{a p r} \underline{r}^{\diamond}(A) \cup \underline{a p r} \underline{\underline{R}}^{\diamond}(B)$, either there is $i$ such that $x \in \underline{R}_{i}^{\prime}(A)$, or there is $j$ such that $x \in \underline{R}_{j}^{\prime}(B)$. Then $x \in s R_{i} \subset A$ or $x \in t R_{j} \subset B$, where $s, t \in U$. However, as $A, B \subseteq A \cup B$, we thus have $x \in \underline{R}_{i}^{\prime}(A \cup B)$ or $x \in \underline{R}_{j}^{\prime}(A \cup B)$. So either way, $x \in \underline{\operatorname{apr}} \underline{v}^{\diamond}(A \cup B)$. Hence the result.

$\left(L_{5}\right)$. Let $A \subseteq B$ and $x \in \underline{a p r} \underline{r}^{\diamond}(A)$. Then $x \in \bigcup_{i=1}^{n} \underline{R}_{i}^{\prime}(A)$, for fixed $i$, we have $x \in s R_{i} \subseteq A$ but $A \subseteq B$. So $x \in s R_{i} \subset A \subseteq B$, hence $x \in \underline{\operatorname{apr}}{ }^{\diamond}(B)$. Thus, $\underline{\operatorname{apr}^{\diamond}}(A) \subseteq \underline{\operatorname{apr}}{ }^{\diamond}(B)$.

$\left(L_{6}\right)$. Since $R_{i}$ are reflexive relations on $U$, then $\forall x \in U$, we have $x \in x R_{i} \quad \forall i=1,2,3, \ldots, n$. So $x R_{i} \not \subset \emptyset$. Thus, $\underline{R}_{i}^{\prime}(\emptyset)=\emptyset \forall i=1,2,3, \ldots, n$. i.e., $\underline{a p r} \underline{r}^{\diamond}(\emptyset)=\emptyset$.

$\left(L_{7}\right)$. As $\underline{\operatorname{apr}}{ }^{\diamond}(A)=\bigcup_{i=1}^{n} \underline{R}_{i}^{\prime}(A)$, but $R^{\prime}(A) \subseteq A \forall i=1,2, \ldots, n$. Then $\underline{\operatorname{apr}}{ }^{\diamond}(A) \subseteq A$.

$\left(L_{9}\right)$. Assume that $y \in \underline{a p r}{ }^{\diamond}(A)$, then there exists $i$ such that $y \in \underline{R}_{i}^{\prime}(A)$. So there is $s \in U$ such that $y \in s R_{i} \subseteq A$. Hence $y \in s R_{i} \subseteq \bigcup_{i=1}^{n} \bigcup\left\{s R_{j}: s R_{j} \subseteq A\right\}$, i.e. $y \in s R_{i} \subseteq \underline{a p r}{ }^{\diamond}(A)$. This implies that $\left.y \in \underline{a p r}{ }^{\diamond} \underline{a p r}{ }^{\diamond}(A)\right)$. Hence the result.

We can prove $U_{2}, U_{4}, U_{5}, U_{6}, U_{7}$ and $U_{9}$ in a manner similar to the proofs of $L_{2}, L_{4}, L_{5}, L_{6}, L_{7}$ and $L_{9}$. We can prove $L U$ from $L_{7}$ and $U_{7}$.

The following example shows that the converse of $L_{4}$ in proposition 4.3 is not true in general.

Example 4.4 Let $R_{1}=\{(a, a),(b, b),(c, c),(d, d),(e, e),(a, b),(a, c),(d, e),(b, d),(c, e),(e, b),(e, a)\}$ and $R_{2}$ $=\{(a, a),(b, b),(c, c),(d, d),(e, e),(a, d),(a, c),(d, a),(b, e),(c, e),(e, d),(e, a)\}$ be any two binary reflexive relations on a non-empty set $U=\{a, b, c, d, e\}$. We have $a R_{1}=\{a, b, c\}, b R_{1}=\{b, d\}, c R_{1}=\{c, e\}, d R_{1}=\{d, e\}, e R_{1}=$ $\{a, b, e\}, a R_{2}=\{a, d, c\}, b R_{2}=\{b, e\}, c R_{2}=\{c, e\}, d R_{2}=\{a, d\}$ and $e R_{2}=\{a, d, e\}$. If $A=\{a, d, e\}$ and $B=\{b, c, d\}$, hence $\underline{R}_{1}^{\prime}(A)=\bigcup\left\{x R_{1}: x R_{1} \subseteq A\right\}=d R_{1}=\{d, e\}, \underline{R}_{2}^{\prime}(A)=\bigcup\left\{x R_{2}: x R_{2} \subseteq A\right\}=e R_{2}=$ $\{a, d, e\}, \underline{R}_{1}^{\prime}(B)=\bigcup\left\{x R_{1}: x R_{1} \subseteq B\right\}=b R_{1}=\{b, d\}, \underline{R}_{2}^{\prime}(B)=\bigcup\left\{x R_{2}: x R_{2} \subseteq B\right\}=\phi \underline{R}_{1}^{\prime}(A \cup B)=$ $\bigcup\left\{x R_{1}: x R_{1} \subseteq A \cup B\right\}=U$ and $\underline{R}_{2}^{\prime}(A \cup B)=\bigcup\left\{x R_{2}: x R_{2} \subseteq A \cup B\right\}=U$. Consequently, apr $\underline{r}^{\diamond}(A)=$ $\underline{R}_{1}^{\prime}(A) \cup \underline{R}_{2}^{\prime}(A)=\{a, d, e\}, \underline{a p r}{ }^{\diamond}(B)=\underline{R}_{1}^{\prime}(B) \cup \underline{R}_{2}^{\prime}(B)=\{b, d\}$ and $\underline{a p r}{ }^{\diamond}(A \cup B)=\underline{R}_{1}^{\prime}(A \cup B) \cup \underline{R}_{2}^{\prime}(A \cup B)=U$. i. e., $\underline{a p r} \underline{r}^{\diamond}(A) \cup \underline{a p r}{ }^{\diamond}(B) \neq \underline{a p r}{ }^{\diamond}(A \cup B)$.

The following example shows that the converse of $U_{4}$ in proposition 4.3 is not true in general.

Example 4.5 In Example 4.4, if $A=\{a, e\}$ and $B=\{c, d\}$, hence $\overline{a p r}^{\diamond}(A)=\{a, c, e\}, \overline{a p r}^{\diamond}(B)=\{c, d\}$ and $\overline{a p r}^{\diamond}(A \cap B)=\emptyset$. Thus $\overline{a p r}^{\diamond}(A) \cap \overline{a p r}^{\diamond}(B) \neq \overline{a p r}^{\diamond}(A \cap B)$.

The following example shows that the converse of $L_{7}, U_{7}$ and $L U$ in proposition 4.3 are not true in general.

Example 4.6 In Example 4.4, if $A=\{a, e\}$, hence $\underline{\operatorname{apr}^{\diamond}}(A)=\emptyset$ and $\overline{a p r}{ }^{\diamond}(A)=\{a, c, e\}$, so $\underline{a p r}^{\diamond}(A) \neq A$, $\overline{a p r}^{\diamond}(A) \neq A$ and $\underline{a p r}^{\diamond}(A) \neq \overline{a p r}^{\diamond}(A)$.

Remark 4.7 Let $R_{i}, i=1,2,3, \ldots, n$ be any reflexive relations, The following properties do not hold, for any subsets $A, B \subseteq U$.

$$
\begin{aligned}
& \left(L_{3}\right) \underline{a p r}^{\diamond}(A \cap B)=\underline{a p r} \underline{r}^{\diamond}(A) \cap \underline{a p r} \underline{r}^{\diamond}(B) . \\
& \left(L_{8}\right) A \subseteq \underline{a p r}{ }^{\diamond}\left(\overline{a p r}^{\diamond}(A)\right) . \\
& \left(L_{10}\right) \overline{a p r}^{\diamond}(A)=\underline{a p r}{ }^{\diamond}\left(\overline{a p r}^{\diamond}(A)\right) .
\end{aligned}
$$




$$
\begin{aligned}
& \left(U_{3}\right) \overline{a p r}^{\diamond}(A \cup B)=\overline{a p r}^{\diamond}(A) \cup \overline{a p r}^{\diamond}(B) . \\
& \left(U_{8}\right) \overline{a p r}^{\diamond}\left(\underline{a p r}{ }^{\diamond}(A)\right) \subseteq A . \\
& \left(U_{10}\right) \overline{a p r}^{\diamond}\left(\underline{a p r}{ }^{\diamond}(A)\right)=\underline{a p r}{ }^{\diamond}(A) . \\
& (C O) \underline{a p r}{ }^{\diamond}\left(A^{c} \cup B\right) \subseteq\left(\underline{a p r}{ }^{\diamond}(A)\right)^{c} \cup \underline{a p r}{ }^{\diamond}(B) .
\end{aligned}
$$

The following examples show Remark 4.7.

Example 4.8 In Example 4.4, if $A=\{b, d\}$ and $B=\{b, e\}$. Hence, $\underline{R}_{1}^{\prime}(A)=\bigcup\left\{x R_{1}: x R_{1} \subseteq A\right\}=b R_{1}=\{b, d\}$, $\underline{R}_{2}^{\prime}(A)=\bigcup\left\{x R_{2}: x R_{2} \subseteq A\right\}=\phi, \underline{R}_{1}^{\prime}(B)=\bigcup\left\{x R_{1}: x R_{1} \subseteq B\right\}=\phi, \underline{R}_{2}^{\prime}(B)=\bigcup\left\{x R_{2}: x R_{2} \subseteq B\right\}=b R_{2}=\{b, e\}$, $\underline{R}_{1}^{\prime}\left(A^{c}\right)=\bigcup\left\{x R_{1}: x R_{1} \subseteq A^{c}\right\}=c R_{1}=\{c, e\}, \underline{R}_{2}^{\prime}\left(A^{c}\right)=\bigcup\left\{x R_{2}: x R_{2} \subseteq A^{c}\right\}=c R_{2}=\{c, e\}, \underline{R}_{1}^{\prime}\left(B^{c}\right)=$ $\bigcup\left\{x R_{1}: x R_{1} \subseteq B^{c}\right\}=\phi, \underline{R}_{2}^{\prime}\left(B^{c}\right)=\bigcup\left\{x R_{2}: x R_{2} \subseteq B^{c}\right\}=a R_{2}=\{a, c, d\}, \underline{R}_{1}^{\prime}(A \cap B)=\bigcup\left\{x R_{1}: x R_{1} \subseteq\right.$ $(A \cap B)\}=\phi, \underline{R}_{2}^{\prime}(A \cap B)=\bigcup\left\{x R_{2}: x R_{2} \subseteq(A \cap B)\right\}=\phi, \underline{R}_{1}^{\prime}\left((A \cup B)^{c}\right)=\bigcup\left\{x R_{1}: x R_{1} \subseteq(A \cup B)^{c}\right\}=\phi$, $\underline{R}_{2}^{\prime}\left((A \cup B)^{c}\right)=\bigcup\left\{x R_{2}: x R_{2} \subseteq(A \cup B)^{c}\right\}=\phi$. Consequently, $\underline{a p r}{ }^{\diamond}(A)=\underline{R}_{1}^{\prime}(A) \cup \underline{R}_{2}^{\prime}(A)=\{b, d\}, \underline{a p r}{ }^{\diamond}(B)=$ $\underline{R}_{1}^{\prime}(B) \cup \underline{R}_{2}^{\prime}(B)=\{b, e\}, \underline{a p r}{ }^{\diamond}\left(A^{c}\right)=\underline{R}_{1}^{\prime}\left(A^{c}\right) \cup \underline{R}_{2}^{\prime}\left(A^{c}\right)=\left\{c, \bar{e}, \underline{a p r}{ }^{\diamond}\left(B^{c}\right)=\underline{R}_{1}^{\prime}\left(B^{c}\right) \cup \underline{R}_{2}^{\prime}\left(B^{c}\right)=\{a, c, d\}\right.$, $\overline{a p r}^{\diamond}(A)=\left(\underline{a p r}^{\diamond}\left(A^{c}\right)\right)^{c}=\{a, b, d\}, \overline{a p r}{ }^{\diamond}(B)=\left(\underline{a p r}^{\diamond}\left(B^{c}\right)\right)^{c}=\{b, e\}, \underline{a p r}{ }^{\diamond}(A \cap B)=\underline{R}_{1}^{\prime}(A \cap B) \cup \underline{R}_{2}^{\prime}(A \cap B)=\phi$, $\left.\underline{a p r}^{\diamond}\left((A \cup B)^{c}\right)=\underline{R}_{1}^{\prime}\left((A \cup B)^{c}\right) \cup \underline{R}_{2}^{\prime}\left((A \cup B)^{c}\right)=\overline{\phi \text { and }} \overline{a p r}{ }^{\diamond}(A \cup B)=\overline{(\operatorname{apr}}{ }^{\diamond}\left((A \cup B)^{c}\right)\right)^{c}=U$. Thus, $\underline{a p r}^{\diamond}(A \cap B) \neq$ $\underline{\underline{a p r}} \diamond(A) \cap \underline{a p r} \underline{v}^{\diamond}(B)$ and $\overline{a p r}{ }^{\diamond}(A) \cup \overline{a p r}^{\diamond}(B) \neq \overline{a p r}^{\diamond}(A \cup B)$. i.e., $L_{3} \overline{\text { and }} U_{3}$ do not hold.

Example 4.9 In Example 4.4, if $A=\{a\}$, hence, $\overline{a p r}^{\diamond}(A)=\{a\}$ and $\underline{a p r}^{\diamond}\left(\overline{a p r}^{\diamond}(A)\right)=\emptyset$. Thus, $A \not \subset \underline{a p r}^{\diamond}($ $\left.\overline{a p r}^{\diamond}(A)\right)$, i.e., $L_{8}$ does not hold.

Example 4.10 In Example 4.4, if $A=\{a, d, e\}$, hence, $\underline{\text { apr }}{ }^{\diamond}(A)=\{a, d, e\}$ and $\overline{a p r}^{\diamond}\left(\underline{\operatorname{apr}}{ }^{\diamond}(A)\right)=U$. So $\overline{a p r}^{\diamond}($ $\left.\operatorname{apr}^{\diamond}(A)\right) \not \subset A$. i.e., $U_{8}$ does not hold.

Example 4.11 In Example 4.4, if $A=\{a, e\}$, hence, $\overline{a p r}^{\diamond}(A)=\{a, c, e\}$ and $\underline{a p r}^{\diamond}\left(\overline{a p r}^{\diamond}(A)\right)=\{c, e\}$. So $\overline{a p r}^{\diamond}(A) \neq \operatorname{apr}^{\diamond}\left(\overline{a p r}^{\diamond}(A)\right)$. i.e., $L_{10}$ does not hold.

Example 4.12 In Example 4.4, if $A=\{b, c, d\}$, hence, $\underline{\operatorname{apr}}{ }^{\diamond}(A)=\{b, d\}$ and $\overline{a p r}^{\diamond}\left(\underline{a p r}{ }^{\diamond}(A)\right)=\{a, b, d\}$, so $\overline{a p r}^{\diamond}($ $\left.\underline{a p r}{ }^{\diamond}(A)\right) \neq \underline{a p r}{ }^{\diamond}(A)$. i.e., $U_{10}$ does not hold.

Example 4.13 In Example 4.4, if $A=\{b, c, d, e\}$ and $B=\{b, c, d\}$, hence, $\underline{\text { apr }}{ }^{\diamond}\left(A^{c} \cup B\right)=\{a, b, c, d\},\left(\underline{a p r}^{\diamond}(A)\right)^{c}=$ $\{a\}$ and $\underline{\operatorname{apr}}{ }^{\diamond}(B)=\{b, d\}$. Thus, $\underline{a p r}{ }^{\diamond}\left(A^{c} \cup B\right) \not \subset\left(\underline{a p r}^{\diamond}(A)\right)^{c} \cup \underline{a p r}{ }^{\diamond}(B)$.i.e., CO does not hold.

Definition 4.14 Let $\left\{R_{i}: i=1,2, \ldots, n\right\}$ be a family of binary relations on a non-empty set $U$, for any set $A \subset U$. We can introduce a definition for n-lower and n-upper approximations of $A$ according to $R_{i}$ as the following:

$$
\underline{a p r}^{\bowtie}(A)=\bigcup_{i=1}^{n} \underline{R}_{i}^{\prime \prime}(A) \quad \text { and } \quad \overline{a p r}^{\bowtie}(A)=\left[\underline{a p r}^{\bowtie}\left(A^{c}\right)\right]^{c}
$$

where $\underline{R}_{i}^{\prime \prime}(A)$ is the lower approximation defined in Definition 3.4.

Notice that the n-upper approximation $\overline{\operatorname{apr}}^{\bowtie}(A)$ defined above is just $\bigcap_{i=1}^{n} \bar{R}_{i}^{\prime \prime}(A)$.

We can define the accuracy measure of any set $A$ according to reflexive relations $R_{i}$, as the following:

$$
\alpha^{\bowtie}(A)=\frac{\left|\underline{a p r}^{\bowtie}(A)\right|}{\left|\overline{\operatorname{apr}^{\bowtie}}(A)\right|} .
$$

Proposition 4.15 For a family of binary reflexive relations $\left\{R_{i}: i=1,2, \ldots, n\right\}$ on a non-empty set $U$ the following properties are hold for every $A, B \subseteq U$ : 


$$
\begin{aligned}
& \left(L_{2}\right) \underline{a p r}{ }^{\bowtie}(U)=U . \\
& \left(L_{4}\right) \underline{a p r}{ }^{\bowtie}(A \cup B) \supseteq \underline{a p r}{ }^{\bowtie}(A) \cup \underline{a p r} \underline{a}^{\bowtie}(B) . \\
& \left(L_{5}\right) A \subseteq B \Longrightarrow \underline{a p r}{ }^{\bowtie}(A) \subseteq \underline{a p r}{ }^{\bowtie}(B) . \\
& \left(L_{6}\right) \underline{a p r}{ }^{\bowtie}(\emptyset)=\emptyset . \\
& \left(L_{7}\right) \underline{a p r}{ }^{\bowtie}(A) \subseteq A . \\
& \left(U_{2}\right) \overline{a p r}{ }^{\bowtie}(\emptyset)=\emptyset . \\
& \left(U_{4}\right) \overline{a p r}{ }^{\bowtie}(A \cap B) \subseteq \overline{a p r}{ }^{\bowtie}(A) \cap \overline{a p r}^{\bowtie}(B) . \\
& \left(U_{5}\right) A \subseteq B \Longrightarrow \overline{a p r}{ }^{\bowtie}(A) \subseteq \overline{a p r}{ }^{\bowtie}(B) . \\
& \left(U_{6}\right) \overline{a p r}{ }^{\bowtie}(U)=U . \\
& \left(U_{7}\right) A \subseteq \overline{a p r}{ }^{\bowtie}(A) . \\
& (L U) \underline{a p r}{ }^{\bowtie}(A) \subseteq \overline{a p r}{ }^{\bowtie}(A) .
\end{aligned}
$$

Proof. $\left(U_{2}\right)$. As $\forall i=1,2, \ldots, n \quad x R_{i} \cap \emptyset=\emptyset$, hence $\overline{a p r}{ }^{\bowtie}(\emptyset)=\emptyset$.

$\left(U_{4}\right)$. Let $x \notin\left(\overline{a p r}^{\bowtie}(A) \cap \overline{a p r}^{\bowtie}(B)\right)$. Then $x \notin \overline{a p r}^{\bowtie}(A)$ or $x \notin \overline{a p r}^{\bowtie}(B)$. We can say that there will be some $i$ for which $x \notin \bar{R}_{i}(A)$, or there will be some $j$ (not necessarily same as $i$ ) for which $x \notin \bar{R}_{j}(B)$. Accordingly, we would have either $x \notin \bar{R}_{i}^{\prime \prime}(A \cap B)$ or $x \notin \bar{R}_{j}^{\prime \prime}(A \cap B)$. Hence the result.

$\left(U_{5}\right)$. Let $A \subseteq B$ and $x \in \overline{a p r}^{\bowtie}(A)$. Then for fixed $i$, we have $x \in s R_{i}$ such that $s R_{i} \cap A \neq \emptyset$, where $s \in U$ but $A \subseteq B$, then $s R_{i} \cap B \neq \emptyset$. So $x \in \bar{R}_{i}^{\prime \prime}(B)$ and so $x \in \overline{a p r}^{\bowtie}(B)$. Thus, $\overline{\operatorname{apr}}^{\bowtie}(A) \subseteq \overline{\operatorname{apr}}^{\bowtie}(B)$.

$\left(U_{6}\right)$. As $R_{i}$ are a reflexive relations, then $\forall x \in U$, we have $x \in x R_{i} \quad \forall i=1,2, \ldots, n$. So $x R_{i} \cap U \neq \emptyset$, this implies that $\overline{a p r}^{\bowtie}(U)=U$.

$\left(U_{7}\right)$. As $R_{i}$ are reflexive relations, then $x \in x R_{i} \quad \forall i=1,2,3, \ldots, n$. So for fixed $i$, we can say that $\forall x \in A$, we have $x \in s R_{i}$ such that $s R_{i} \cap A \neq \emptyset$, this implies that $x \in \bar{R}_{i}(A)$. Thus, $x \in \overline{a p r}^{\bowtie}(A)$. i.e., $A \subset \overline{a p r}^{\bowtie}(A)$. We can prove $L_{2}, L_{4}, L_{5}, L_{6}$ and $L_{7}$ as the same as $U_{2}, U_{4}, U_{5}, U_{6}$ and $U_{7}$. We can prove $L U$ from $L_{7}$ and $U_{7}$.

The following example shows that the converse of $L_{4}$ in Proposition 4.15 is not true in general.

Example 4.16 In Example 4.4, if $A=\{b\}$ and $B=\{e\}$, hence $\underline{a p r}^{\bowtie}(A)=\emptyset$, $\underline{a p r}^{\bowtie}(B)=\emptyset$ and $\underline{a p r}{ }^{\bowtie}(A \cup B)=\{b\}$. $i, . e ., \underline{a p r}^{\bowtie}(A) \cup \underline{a p r}{ }^{\bowtie}(B) \neq \underline{a p r}^{\bowtie}(A \cup B)$.

The following example shows that the converse of $U_{4}$ in Proposition 4.15 is not true in general.

Example 4.17 In Example 4.4, if $A=\{a\}$ and $B=\{b\}$, hence $\overline{a p r}^{\bowtie}(A)=\{a, c, e\}, \overline{a p r}^{\bowtie}(B)=\{b, e\}$ and $\overline{a p r}^{\bowtie}(A \cap B)=\emptyset$. Thus $\overline{a p r}{ }^{\bowtie}(A) \cap \overline{a p r}^{\bowtie}(B) \not \subset \overline{a p r}^{\bowtie}(A \cap B)$.

The following example shows that the converse of $L_{7}, U_{7}$ and $L U$ in Proposition 4.15 are not true in general.

Example 4.18 In Example 4.4, if $A=\{a, c\}$, hence $\underline{a p r}^{\bowtie}(A)=\emptyset$ and $\overline{a p r}^{\bowtie}(A)=\{a, c, e\}$, so apr $\underline{a p}^{\bowtie}(A) \not \supset A$, $\overline{a p r}^{\bowtie}(A) \not \subset A$ and $\underline{a p r}^{\bowtie}(A) \not \supset \overline{a p r}{ }^{\bowtie}(A)$.

Remark 4.19 Let $R_{i}, i=1,2, \ldots, n$ be any reflexive relations. The following properties do not hold for all subsets $A, B$ of $U$.

$$
\begin{aligned}
& \left(L_{3}\right) \underline{a p r^{\bowtie}}(A \cap B)=\underline{a p r} r^{\bowtie}(A) \cap \underline{a p r}{ }^{\bowtie}(B) . \\
& \left(L_{8}\right) A \subseteq \underline{a p r}{ }^{\bowtie}\left(\overline{a p r}{ }^{\bowtie}(A)\right) . \\
& \left(L_{9}\right) \underline{a p r}{ }^{\bowtie}\left(\underline{a p r}{ }^{\bowtie}(A)\right)=\underline{a p r}{ }^{\bowtie}(A) .
\end{aligned}
$$




$$
\begin{aligned}
& \left(L_{10}\right) \overline{a p r}^{\bowtie}(A)=\underline{a p r} r^{\bowtie}\left(\overline{a p r}^{\bowtie}(A)\right) . \\
& \left(U_{3}\right) \overline{a p r}{ }^{\bowtie}(A \cup B)=\overline{a p r}{ }^{\bowtie}(A) \cup \overline{a p r}{ }^{\bowtie}(B) . \\
& \left.\left(U_{8}\right) \overline{a p r} r^{\bowtie} \underline{\operatorname{apr}}{ }^{\bowtie}(A)\right) \subseteq A . \\
& \left(U_{9}\right) \overline{a p r}{ }^{\bowtie}\left(\overline{a p r}{ }^{\bowtie}(A)\right)=\overline{a p r}{ }^{\bowtie}(A) . \\
& \left(U_{10}\right) \overline{a p r}{ }^{\bowtie}\left(\underline{a p r}^{\bowtie}(A)\right)=\underline{a p r}^{\bowtie}(A) . \\
& (C O) \underline{a p r}{ }^{\bowtie}\left(A^{c} \cup B\right) \subseteq\left(\underline{a p r}^{\bowtie}(A)\right)^{c} \cup \underline{a p r}^{\bowtie}(B) .
\end{aligned}
$$

The following examples show Remark 4.19.

Example 4.20 In Example 4.4, if $A=\{a, c, d, e\}$ and $B=\{b, c, d, e\}$, hence $\underline{a p r}^{\bowtie}(A)=\{a, c, d\}, \underline{a p r}^{\bowtie}(B)=\{b, d\}$ and $\underline{a p r}^{\bowtie}(A \cap B)=\emptyset$. Thus $\underline{a p r}{ }^{\bowtie}(A \cap B) \not \supset \underline{a p r}^{\bowtie}(A) \cap \underline{a p r}^{\bowtie}(B)$. Consequently, $\underline{a p r}^{\bowtie}(A \cap B) \neq \underline{a p r} \underline{ }^{\bowtie}(A) \cap \underline{a p r}^{\bowtie}(B)$, i.e., $\overline{L_{3}}$ does not hold.

Example 4.21 In Example 4.4, if $A=\{a\}$ and $B=\{b\}$, then $\overline{a p r}^{\bowtie}(A)=\{a, c, e\}, \overline{a p r}^{\bowtie}(B)=\{b, e\}$ and $\overline{\operatorname{apr}}^{\bowtie}(A \cup B)=U$. Thus, $\overline{\operatorname{apr}}{ }^{\bowtie}(A) \cup \overline{\operatorname{apr}}^{\bowtie}(B) \not \supset \overline{\operatorname{apr}}^{\bowtie}(A \cup B)$. Consequently, $\overline{\operatorname{apr}}^{\bowtie}(A) \cup \overline{\operatorname{apr}^{\bowtie}}(B) \neq \overline{\operatorname{apr}}^{\bowtie}(A \cup B)$, i.e., $U_{3}$ does not hold.

Example 4.22 In Example 4.4, if $A=\{a\}$, hence $\overline{a p r}^{\bowtie}(A)=\{a, c, e\}$ and $\underline{a p r}^{\bowtie}\left(\overline{a p r}^{\bowtie}(A)\right)=\emptyset$. Thus $A \not \subset \underline{a p r}^{\bowtie}($ $\left.\overline{\operatorname{apr}}^{\bowtie}(A)\right)$, i.e., $L_{8}$ does not hold.

Example 4.23 In Example 4.4, if $A=\{b, c, d, e\}$, hence $\underline{a p r}^{\bowtie}(A)=\{b, d\}$ and $\overline{a p r}^{\bowtie}\left(\underline{a p r} r^{\bowtie}(A)\right)=U$. So $\overline{a p r}^{\bowtie}($

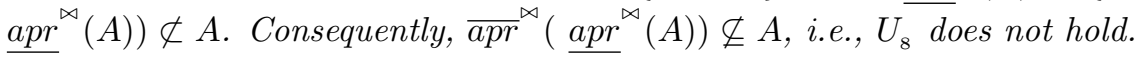

Example 4.24 In Example 4.4, if $A=\{a, b, c, e\}$, hence $\underline{a p r}^{\bowtie}(A)=\{a, b, c\}$ and $\underline{a p r}^{\bowtie}\left(\underline{a p r}^{\bowtie}(A)\right)=\emptyset$, so $\left.\underline{\operatorname{apr}}^{\bowtie}(A) \not \subset \underline{\operatorname{apr}} \underline{a p}^{\bowtie} \underline{a p r}^{\bowtie}(A)\right)$.

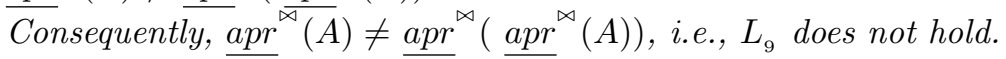

Example 4.25 In Example 4.4, if $A=\{d\}$, hence $\overline{a p r}^{\bowtie}(A)=\{d, e\}$ and $\overline{a p r}^{\bowtie}\left(\overline{a p r}^{\bowtie}(A)\right)=U$. So $\overline{a p r}^{\bowtie}(A) \not \supset$ $\overline{a p r}^{\bowtie}\left(\overline{\operatorname{apr}}{ }^{\bowtie}(A)\right)$.

Consequently, $\overline{a p r}^{\bowtie}(A) \neq \overline{a p r}^{\bowtie}\left(\overline{a p r}^{\bowtie}(A)\right)$, i.e., $U_{9}$ does not hold.

Example 4.26 In Example 4.4, if $A=\{a\}$, hence $\overline{a p r}^{\bowtie}(A)=\{a, c, e\}$ and $\underline{a p r}^{\bowtie}\left(\overline{a p r}^{\bowtie}(A)\right)=\emptyset$. So $\overline{a p r}^{\bowtie}(A) \not \subset$ $\underline{a p r}^{\bowtie}\left(\overline{a p r}^{\bowtie}(A)\right)$.

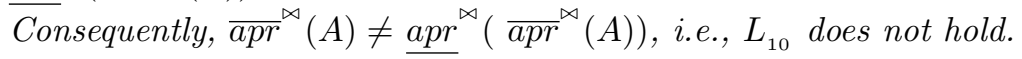

Example 4.27 In Example 4.4, if $A=\{a, b, c, e\}$, hence $\underline{a p r}^{\bowtie}(A)=\{a, b, c\}$ and $\overline{a p r}^{\bowtie}\left(\underline{a p r}^{\bowtie}(A)\right)=U$, so $\overline{a p r}^{\bowtie}($ $\left.\underline{a p r}^{\bowtie}(A)\right) \not \subset \underline{a p r}^{\bowtie}(A)$. Consequently, $\overline{\operatorname{apr}^{\bowtie}}\left(\underline{\operatorname{apr}}^{\bowtie}(A)\right) \neq \underline{\operatorname{apr}^{\bowtie}}(A)$, i.e., $U_{10}$ does not hold.

Example 4.28 In Example 4.4, if $A=\{a, c, d, e\}$ and $B=\{a, c, e\}$, hence $\left(\underline{a p r}^{\bowtie}(A)\right)^{c}=\{b, e\}$, apr ${ }^{\bowtie}(B)=\emptyset$ and $\underline{a p r}{ }^{\bowtie}\left(A^{c} \cup B\right)=\{a, b, c\}$. Thus $\left(\underline{\operatorname{apr}}{ }^{\bowtie}(A)\right)^{c} \cup \underline{\operatorname{apr}}{ }^{\bowtie}(B) \not \supset \underline{\operatorname{apr}}{ }^{\bowtie}\left(A^{c} \cup B\right)$. i.e., $C O$ does not hold.

For a family of reflexive relations $R_{i}, i=1,2, \ldots, n$ on a non-empty set $U$, the comparison between the properties of rough sets depends on Definition 4.1 and Definition 4.14 as shown in the following table.

\section{Comparison between all these lower and upper approximations}

In this section, we will show the available relations between some of these approximations. 
Table 2: Upper and lower approximations under reflexive relation by using Definition 4.1 and Definition 4.14

\begin{tabular}{ccc}
\hline Properties & Definition 4.1 & Definition 4.14 \\
\hline$L_{1}$ & $\star$ & $\star$ \\
$L_{2}$ & $\star$ & $\star$ \\
$L_{3}$ & & $\star$ \\
$L_{4}$ & $\star$ & $\star$ \\
$L_{5}$ & $\star$ & $\star$ \\
$L_{6}$ & $\star$ & $\star$ \\
$L_{7}$ & $\star$ & \\
$L_{8}$ & & \\
$L_{9}$ & & \\
$L_{10}$ & & $\star$ \\
$U_{1}$ & $\star$ & \\
$U_{2}$ & $\star$ & $\star$ \\
$U_{3}$ & & $\star$ \\
$U_{4}$ & $\star$ & $\star$ \\
$U_{5}$ & $\star$ & $\star$ \\
$U_{6}$ & $\star$ & \\
$U_{7}$ & $\star$ & \\
$U_{8}$ & & \\
$U_{9}$ & & \\
$U_{10}$ & & \\
$C O$ & & \\
$L U$ & &
\end{tabular}

\subsection{For one user}

Let $R$ be any reflexive relation on a non-empty set $U$ and $A \subset U$, we have

$$
\underline{R}^{\prime \prime}(A) \subset \underline{R}(A) \subset \underline{R^{\prime}}(A) \subset A \subset \bar{R}^{\prime}(A) \subset \bar{R}(A) \subset \bar{R}^{\prime \prime}(A) .
$$

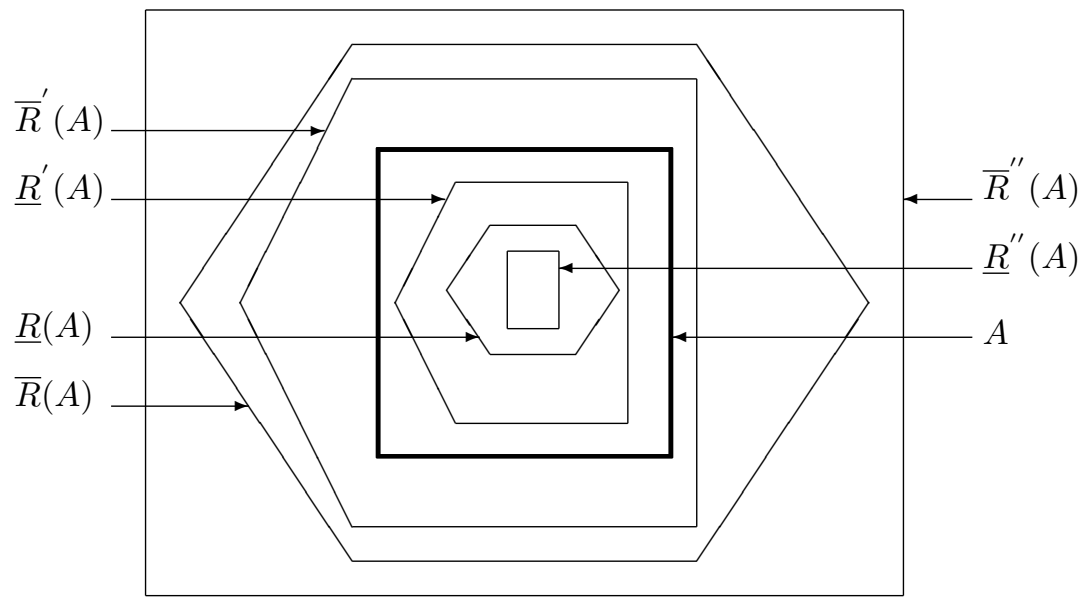

Figure 1

From Figure 1, we can note that the definitions of lower and upper approximations divide the boundary region into 6-regions. By using Definition 3.3 for lower and upper approximations, we get the best lower and upper approximations $A$ with respect to one reflexive relation. We have:

$$
0 \leq \alpha^{\prime \prime}(A) \leq \alpha(A) \leq \alpha^{\prime}(A) \leq 1
$$


This means that, by using Definition 3.3, as a lower and upper approximations for any set $A \subset U$ according to one reflexive relation $R$, set $A$ will be more accurate than the other two definitions, as shown in the following example.

Example 5.1 In Example 4.4, if $A=\{a, c, e\}$, hence $\underline{R}_{1}^{\prime \prime}(A)=\emptyset, \underline{R}_{1}(A)=\{c\}, \underline{R}_{1}^{\prime}(A)=\{c, e\}, \bar{R}_{1}^{\prime \prime}(A)=U$, $\bar{R}_{1}(A)=\{a, c, d, e\}$ and $\bar{R}_{1}^{\prime}(A)=\{a, c, e\}$. Then we have

$$
\underline{R}_{1}^{\prime \prime}(A) \subset \underline{R}_{1}(A) \subset \underline{R}_{1}^{\prime}(A) \subset A \subset \bar{R}_{1}^{\prime}(A) \subset \bar{R}_{1}(A) \subset \bar{R}_{1}^{\prime \prime}(A) .
$$

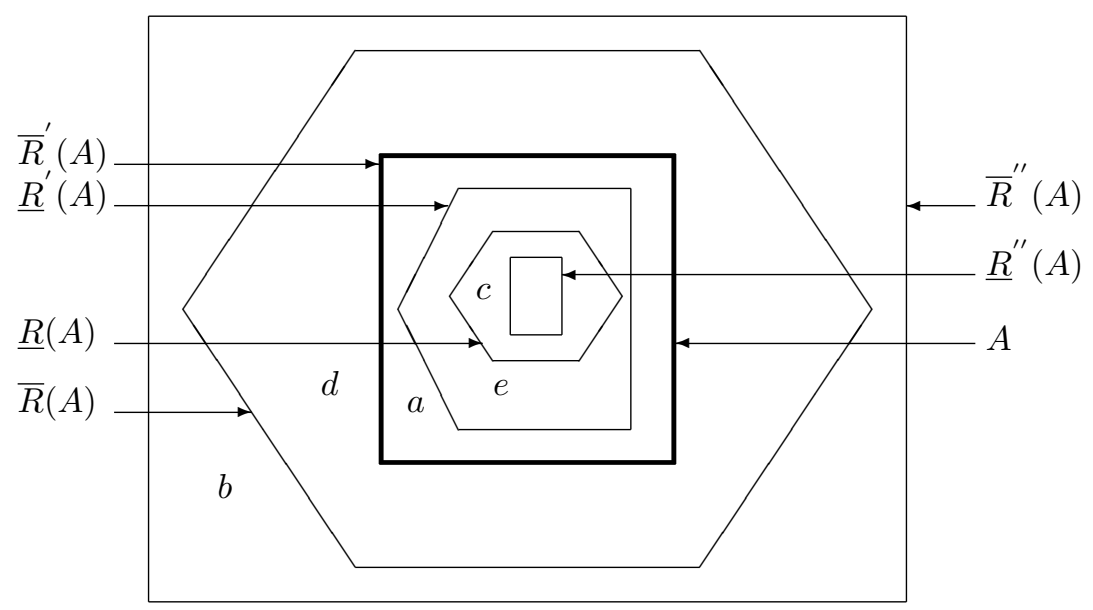

Figure 2

We can also determine the values of the accuracy measure of $A$ by using these definitions, as the following results: $\alpha_{1}^{\prime \prime}(A)=0, \alpha_{1}(A)=1 / 4$ and $\alpha_{1}^{\prime}(A)=2 / 3$. i.e., the degree of the exactness of $A$ by using Definition 3.4 equals $(0 \%)$, which means that $A$ is a completely rough set. By using Definition 3.2, the degree of the exactness of $A$ equals (25\%), which means that $A$ is a rough set by a ratio of $(75 \%)$. And by using Definition 3.3 , the degree of exactness of $A$ equals nearly $(66.7 \%)$, which means that $A$ is a rough set by ratio of nearly $(33.3 \%)$.

It has to be noted that Definition 3.3, has the best approximations for any set $A$ according to one reflexive relation.

\subsection{For n-users: by using the new kind of generalization}

Let $\left\{R_{i}: i=1,2, \ldots, n\right\}$ be a finite family of reflexive relations on a non-empty set $U$, for any set $A \subset U$, we generalized the approximations defined in Definition 3.3 and Definition 3.4, by Definition 4.1 and Definition 4.14 respectively, by using the intersection and the union of upper and lower approximations defined in Definitions 3.2, 3.3 and 3.4. As $\forall i=1,2, \ldots, n \underline{R}_{i}^{\prime}(A) \subset \underline{\operatorname{apr}}{ }^{\diamond}(A), \underline{R}_{i}^{\prime \prime}(A) \subset \underline{a p r}{ }^{\bowtie}(A), \overline{\operatorname{apr}}^{\diamond}(A) \subset \bar{R}_{i}^{\prime}(A)$ and $\overline{a p r}^{\bowtie}(A) \subset \bar{R}_{i}^{\prime \prime}(A)$. But we have

$$
\underline{R}^{\prime \prime}(A) \subset \underline{R}(A) \subset \underline{R^{\prime}}(A) \subset A \subset \bar{R}^{\prime}(A) \subset \bar{R}(A) \subset \bar{R}^{\prime \prime}(A)
$$

This implies that

$$
\underline{\operatorname{apr}}^{\bowtie}(A) \subset \underline{\operatorname{apr}}^{\diamond}(A) \subset A \subset \overline{\operatorname{apr}}^{\diamond}(A) \subset \overline{a p r}^{\bowtie}(A) .
$$

We can note that:

$$
\underline{R}^{\prime \prime}(A) \subset \underline{R}(A) \subset \underline{R}^{\prime}(A) \subset \underline{a p r} \underline{r}^{\diamond}(A) \subset A \subset \overline{a p r}^{\diamond}(A) \subset \bar{R}^{\prime}(A) \subset \bar{R}(A) \subset \bar{R}^{\prime \prime}(A) .
$$




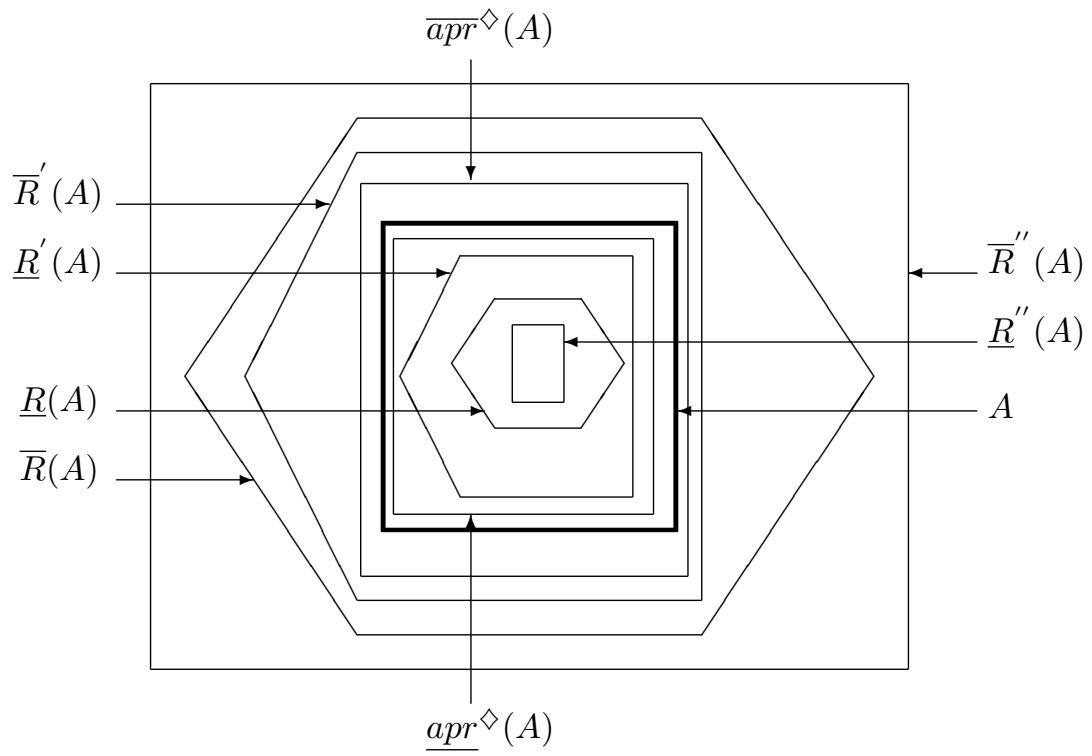

Figure 3

From Figure 3, we can note that these definitions of lower and upper approximations divide the boundary region into 8-regions. By using Definition 4.1, for $n$-lower and $n$-upper approximations, we get the best approximation for any set $A \subset U$ according to a finite number of reflexive relations because we divided the boundary region (indicated by using Definition 3.3, which is the best of the last approximations) into three regions by using the generalized approximations defined in Definition 4.1, i.e., we decreased the boundary region more and more. So Definition 4.1, has the best approximations. This leads to:

$$
0 \leq \alpha^{\prime \prime}(A) \leq \alpha(A) \leq \alpha^{\prime}(A) \leq \alpha^{\diamond}(A) \leq 1
$$

This means that, by using approximations defined in Definition 4.1, any non-empty set $A \subset U$ will be more exact than any other approximations.

The following examples show that.

Example 5.2 In Example 4.4, if $A=\{a, b, d\}$, hence $\underline{R}_{1}^{\prime \prime}(A)=\emptyset, \underline{R}_{1}(A)=\{b\}, \underline{R}_{1}^{\prime}(A)=\{b, d\}, \bar{R}_{1}^{\prime \prime}(A)=U$, $\bar{R}_{1}(A)=\{a, b, d, e\}, \bar{R}_{1}^{\prime}(A)=\{a, b, d\}, \underline{a p r}^{\bowtie}(A)=\emptyset, \underline{a p r}{ }^{*}(A)=\{b, d\}, \underline{a p r}{ }^{\diamond}(A)=\{a, b, d\}, \overline{a p r}^{\bowtie}(A)=U$, and $\overline{\operatorname{apr}}^{\diamond}(A)=\{a, b, d\}$. So, we have:

$$
\underline{a p r}^{\bowtie}(A) \subset \underline{a p r} \underline{v}^{\diamond}(A) \subset A \subset \overline{a p r}^{\diamond}(A) \subset \overline{a p r}^{\bowtie}(A) .
$$

We also get:

$$
\underline{R}_{1}^{\prime \prime}(A) \subset \underline{R}_{1}(A) \subset \underline{R}_{1}^{\prime}(A) \subset \underline{a p r} \underline{g}^{\diamond}(A) \subset A \subset \overline{a p r}^{\diamond}(A) \subset \bar{R}_{1}^{\prime}(A) \subset \bar{R}_{1}(A) \subset \bar{R}_{1}^{\prime \prime}(A) .
$$

Example 5.3 In Example 5.2, we have $\alpha_{1}^{\prime \prime}(A)=0, \alpha_{1}(A)=1 / 4, \alpha_{1}^{\prime}(A)=2 / 3$ and $\alpha^{\diamond}(A)=1$. This implies that:

$$
0 \leq \alpha^{\prime \prime}(A) \leq \alpha(A) \leq \alpha^{\prime}(A) \leq \alpha^{\diamond}(A) \leq 1
$$

This means that, by using the approximations defined in Definition 3.4, the degree of exactness of $A$ equals $0 \%$, so $A$ is completely rough set. The degree of exactness of $A$ by using the approximations defined in Definition 3.2, equals $25 \%$, so $A$ is rough with a ratio of $75 \%$, by using the approximations defined in Definition 3.3 , the degree of exactness of $A$ equals nearly $67 \%$, so $A$ is rough with a ratio of nearly $33 \%$. the degree of exactness of $A$ by using the approximations defined in Definition 4.1 equals to $100 \%$, so $A$ is completely exact set. So the n-lower and n-upper approximations defined in Definition 4.1, is the best one among all these definitions.

We illustrate the following real life example to show the importance of our approximations. 
Table 3:

\begin{tabular}{cccccccc}
\hline & $a_{1}$ & $a_{2}$ & $a_{3}$ & $a_{4}$ & $a_{5}$ & $a_{6}$ & $a_{7}$ \\
\hline 1 & 0.23 & 0.31 & -0.55 & 254.2 & 2.126 & -0.02 & 82.2 \\
2 & -0.48 & -0.60 & 0.51 & 303.6 & 2.994 & -1.24 & 112.3 \\
3 & -0.61 & -0.77 & 1.20 & 287.9 & 2.994 & -1.08 & 103.7 \\
4 & 0.45 & 1.54 & -1.40 & 282.9 & 2.933 & -0.11 & 99.1 \\
5 & -0.11 & -0.22 & 0.29 & 335.0 & 3.458 & -0.19 & 127.5 \\
6 & -0.51 & -0.64 & 0.76 & 311.6 & 3.243 & -1.43 & 120.5 \\
7 & 0.00 & 0.00 & 0.00 & 224.9 & 1.662 & 0.03 & 65.0 \\
8 & 0.15 & 0.13 & -0.25 & 337.2 & 3.856 & -1.06 & 140.6 \\
9 & 1.20 & 1.80 & -2.10 & 322.6 & 3.350 & 0.04 & 131.7 \\
10 & 1.28 & 1.70 & -2.00 & 324.0 & 3.518 & 0.12 & 131.5 \\
\hline
\end{tabular}

Table 4:

\begin{tabular}{ccccc}
\hline$i$ & $\underline{R}_{i}^{\prime}(A)$ & $\bar{R}_{i}^{\prime}(A)$ & $\underline{a_{p r}}{ }^{\diamond}(A)$ & $\overline{a p r^{\diamond}}(A)$ \\
\hline 1 & $\phi$ & $\{1,2,3,5,6,7,8\}$ & & \\
2 & $\phi$ & $\{1,2,3,4,5,6,7,8\}$ & & \\
3 & $\{3,6\}$ & $U$ & $\{3,6,8\}$ & $\{2,3,5,6,8\}$ \\
4 & $\phi$ & $U$ & & \\
5 & $\{8\}$ & $U$ & & \\
6 & $\phi$ & $\{2,3,5,6,8\}$ & & \\
7 & $\phi$ & $U$ & & \\
\hline
\end{tabular}

Example 5.4 Let $U=\{1,2,3,4,5,6,7,8,9,10\}$ be ten amino acids (AAs). The (AAs) are described in terms of seven attributes: $a_{1}=P I E$ and $a_{2}=P I F$ (two measures of the side chain lipophilicity), $a_{3}=D G R=\triangle G$ of transfer from the protein interior to water, $a_{4}=S A C=$ surface area, $a_{5}=M R=$ molecular refractivity, $a_{6}=L A M=$ the side chain polarity, and $a_{7}=$ Vol $=$ molecular volume ([11]).

Table 3 shows all quantitative attributes of ten AAs. Consider seven reflexive relations on $U$ defined as follows:

$$
R_{i}=\left\{(x, y): x\left(a_{i}\right)-y\left(a_{i}\right)<\frac{\sigma_{i}}{2}\right\} \quad \forall x, y \in U
$$

where $\sigma_{i}$ represents the standard deviation of the quantitative attributes $a_{i}, i=1,2, \ldots, 7$. If we take $A=\{3,5,6,8\}$ be a set containing four AAs which have a property $P$.

Table 4 shows the comparison between the lower (resp. upper) approximation $\underline{R}_{i}^{\prime}(A)\left(\operatorname{resp} . \bar{R}_{i}^{\prime}(A)\right)$ according to Yao and our lower (resp. upper) approximation which is defined in Definition 4.1. Notice in this example that $\underline{\operatorname{apr}}^{\bowtie}(A)=\underline{R}_{i}^{\prime \prime}(A)=\phi$ and $\overline{a p r}{ }^{\bowtie}(A)=\bar{R}_{i}^{\prime \prime}(A)=U \forall i=1,2,3, \ldots, 7$.

\section{Conclusion}

In this paper we studied two definitions for lower and upper approximations of any set with respect to one reflexive relation. We also studied the relationship between these types of lower and upper approximations. We introduced a generalization for these definitions by using a finite number of reflexive relations based on the intersection and union of upper and lower approximations of the same set with respect to every relation of them. Also, we compared between these generalized definitions and the last two definitions rather than determining there the best generalized definition as the boundary region is decreased by this definition more than by any other definition. All these results are shown in tables and diagrams. 


\section{Acknowledgements}

The authors would like to thank the anonymous referees for their valuable suggestions in improving this paper.

\section{References}

[1] H. M. Abu-Donia, "Multi Knowledge based rough approximations and applications", Knowledge-Based systems, Vol.26, No.X, (2012), pp.20-29.

[2] H.M. Abu-Donia and A.S. Salama, "Generalization of Pawlak's rough approximation spaces by using $\delta \beta$-open sets", International Journal of Approximate Reasoning, Vol.53, (2012), pp.1094-1105.

[3] H. M. Abu-Donia, "New Rough Set Approximation Spaces", Abstract and Applied Analysis, Vol.2013, (2013), pp.1-7.

[4] H.M. Abu-Donia, "Comparison between different kinds of approximations by using a family of binary relations", Knowledge-Based Systems, Vol.21, (2008), pp.911-919.

[5] M. Banerjee and M.K. Chakraborty, "Rough consequence and rough algebra. In: Rough Sets, Fuzzy Sets and Knowledge Discovery", Proc. Int. Workshop on Rough Sets and Knowledge Discovery (RSKD '93), Banff, Canada 1993, Ed. Ziarko, W.P. (London: Springer-Verlag), (1994), pp.196-207.

[6] G. Cattaneo, D. Ciucci, "Algebraic structures for rough sets", LNCS, Vol.3135, (2004), pp.208-252.

[7] G. Cattaneo, "Abstract approximation spaces for rough theories", in: L. Polkowski, A. Skowron (Eds.), Physica-Verlag, Heidelberg, , (Chapter 4) Vol.1, No.X, (1998), pp.59-98.

[8] G. Cattaneo, "Generalized rough sets (preclusivity fuzzy intuitionstic (BZ)lattice)", studia logica, Vol.53, (1997), pp.47-77.

[9] M. Chuchro, "On rough sets in topological Boolean algebras, in: W. Ziarko (Ed.)", Rough Sets, Fuzzy Sets and Knowledge Discovery, Springer-Verlag, Berlin, (1994), pp.157-160.

[10] S. Comer, "An algebraic approach to the approximation of information", Fundamenta Informaticae, Vol.14, (1991), pp.492-502.

[11] N.E. Tayar, R.S. Tsai, P.A. Carrupt, B. Testa, "Octan-1-ol-water partition coefficients of zwitterionic $\alpha$-amino acid, determination by centrifugal partition chromatography and factorization into steric/hydrophobic and polar components", J. Chem. Soc., Perkin. Trans., Vol.2, (1992), pp.79-84.

[12] P. Eklund, M.A. Galan, Werner Gahler, "Partially Ordered Monads for Monadic Topologies, Rough Sets and Kleene Algebras", Electronic Notes in Theoretical Computer Science, Vol.225, No.2, (2009), pp.67-81.

[13] T. P. Hong, Y. L. Liou and S. L. Wang, "Fuzzy rough sets with hierarchical quantitative attributes", Expert Systems with Applications, Vol.36, No.3, (2009), pp.6790-6799.

[14] M. A. Khan, and M. Banerjee, "Formal reasoning with rough sets in multiple-source approximation systems", Int. J. Approximate Reasoning, Vol.49, No.2, (2008), pp.466-477.

[15] M. Kondo, "On the Structure of Generalized Rough Sets", Information Sciences, Vol.176, No.5, (2005), pp.589-600.

[16] J. Kortelainen, "On Relationship between modified sets, topological space and rough sets", On Relationship between modified sets, Vol.61, (1994), pp.91-95.

[17] T.Y. Lin, Q. Liu, "Rough approximate operators: axiomatic rough set theory", in: W. Ziarko (Ed.), Rough Sets, Fuzzy Sets and Knowledge Discovery, Springer, Berlin, (1994), pp.256-260.

[18] G. Liu and Y. Sai, "A comparison of two types of rough sets induced by coverings", International Journal of Approximate Reasoning, Vol.50, (2009), pp.521-528.

[19] J.-S. Mi, W.-X. Zhang, "An axiomatic characterization of a fuzzy generalization of rough sets", Information Sciences, Vol.160, No.(1-4), (2004), pp.235-249.

[20] J.N. Mordeson, "Rough set theory applied (fuzzy) ideal to theory", Fuzzy Sets and Systems, Vol.121, (2001), pp.315-324.

[21] E. Orlowska, "Semantics analysis of inductive reasoning", Theoretical Computer Science, Vol.43, (1986), pp.81-89.

[22] Z. Pawlak, A. Skowron, "Rough sets: Some extensions", Information Sciences, Vol.177, (2007), pp.28-40. 
[23] Z. Pawlak, A. Skowron, "Rudiments of rough sets", Journal of Information Sciences, Vol.177, (2007), pp.3-27.

[24] Z. Pawlak, A. Skowron, "rough sets and Boolean reasoning", Journal of Information Sciences, Vol.177, (2007), pp.41-73.

[25] Z. Pawlak, "Rough Sets: Theoretical Aspects of Reasoning about Data, System Theory, Knowledge Engineering and Problem Solving", Kluwer Academic Publishers, Dordrecht, The Netherlands, Vol.9, (1991).

[26] Z. Pawlak, "Rough Probability", Bull Polish Acad Sci, Vol.32, (1984), pp.607-612.

[27] Z. Pawlak, "Rough sets", International Journal of Computer and Information Sciences, Vol.11, (1982), pp.341-356.

[28] Z. Pawlak, "Information systems, Theoretical Foundations", Information Systems, Vol.6, (1981), pp.205-218.

[29] J.A. Pomykala, "Approximation operations in approximation space", Bulletin of the Polish Academy of Sciences: Mathematics, Vol.35, (1987), pp.653-662.

[30] K. Qin, Z. Pei, "On the topological properties of fuzzy rough sets", Fuzzy Sets and Systems, Vol.151, No.3, (2005), pp.601-613.

[31] Y. Qian, J. Liang, Y. Yao, C. Dang "MGRS: A multi-granulation rough set", Information Sciences, Vol.180, (2010), pp.949-970.

[32] E.A. Rady, A.M. Kozae, M.M.E. Abd El-Monsef, "Generalized rough sets", Chaos, Solitons and Fractals, Vol.21, (2004), pp.49-53.

[33] C. Rauszer, "Rough logic for multi-agent systems", In: Logic at Work 1992. LNCS (LNAI) Springer, Heidelberg, Vol.808, (1994), pp.161-181.

[34] R. Slowinski, D. Vanderpooten, "A generalized definition of rough approximations based on similarity", EEE Transactions on Knowledge and Data Engineering, Vol.12, No.2, (2000), pp.331-336.

[35] R. Slowinski, D. Vanderpooten, "Similarity relation as a basis for rough approximations", in: P.P. Wang (Ed.), Advances in Machine Intelligence and Soft-Computing, Department of Electrical Engineering, Duke University, Durham, NC, $U S A$, , (1997), pp.17-33.

[36] A. Skowron, "Rough sets and vague concepts", Fundamenta Informaticae, Vol.64, No.1-4, (2005), pp.417-431.

[37] J. Stepaniuk and A. Skowron, "Tolerance approximation spaces, Fundamenta Informaticae", journal, Vol.27, (1996), pp.245-253.

[38] H. Thiele, "On axiomatic characterisations of crisp approximation operators", Information Sciences, Vol.129, (2000), pp.221-226.

[39] Wei-Hua Xu and Wen-Xiu Zhang, "Measuring roughness of generalized rough sets induced by a covering", Fuzzy Sets and Systems, Vol.158, (2007), pp.2443 - 2455.

[40] W.-Z. Wu, Y. Leung, J.-S. Mi, "On characterizations of (I,T)-fuzzy rough approximation operators ", Fuzzy Sets and Systems, Vol.154, No.1, (2005), pp.76-102.

[41] Y. Yao, "On generalizing Pawlak approximation operators", in: LNAI, Vol.1424, (1998), pp.298-307.

[42] Y. Yao, "Relational interpretations of neighborhood operators and rough set approximation operators", Information Sciences, Vol.101, (1998), pp. 239-259.

[43] Y.Y. Yao, "Constructive and algebraic methods of the theory of rough sets", Journal of Information Sciences, Vol.109, (1998), pp.21-47.

[44] Y. Y. Yao, "Generalized rough set models", in: Rough Sets in Knowledge Discovery, Polkowski, L. and Skowron, A. (Eds.), Physica-Verlag, Heidelberg, V (1998), pp.286-318.

[45] Y.Y. Yao, "Two views of the theory of rough sets in finite universes", International Journal of Approximate Reasoning, Vol.15, (1996), pp.291-317.

[46] Y.Y. Yao, T.Y. Lin, "Generalization of rough sets using modal logic, Intelligent Automation and Soft Computing", an International Journal, Vol.2, (1996), pp.103-120.

[47] W. Zakowski, "On a concept of rough sets", Demonstratio Mathematica, Vol.XV, (1982), pp.1129-1133. 
[48] H. P. Zhang, Y. Ouyang, Zhudeng Wang, "Note on Generalized rough sets based on reflexive and transitive relations", Information Sciences, Vol.179, (2009), pp.471-473.

[49] W. Zhu, "Relationship among basic concepts in covering-based rough sets", Information Sciences, Vol.179, (2009), pp. 2478-2486.

[50] W. Zhu, "Relationship between generalized rough sets based on binary relation and covering", Information Sciences, Vol.179 No. (3) 16 , (2009), pp.210-225.

[51] W. Zhu, "Topological approaches to covering rough sets", Information Sciences, Vol.177, No.6, (2007), pp.1499-1508.

[52] W. Zhu, F.-Y. Wang, "On three types of covering rough sets", IEEE Transactions on Knowledge and Data Engineering, Vol.19, No.8, (2007), pp.1131-1144.

[53] W. Zhu, F.-Y. Wang, "Relationships among three types of covering rough sets", in: IEEE GrC, (2006), pp.43-48.

[54] W. Zhu, F.-Y. Wang, "Axiomatic systems of generalized rough sets", in: RSKT 2006, LNAI, Vol.4062, (2006), pp.216-221.

[55] W. Zhu, F.-Y. Wang, "A new type of covering rough sets", in: IEEE IS 2006, Vol.4-6, (2006), pp.444-449.

[56] W. Zhu, F.-Y. Wang, "Binary relation based rough sets", in: IEEE FSKD 2006, LNAI, Vol.4223, (2006), pp.276-285.

[57] W. Ziarko, (ed.), "Rough sets, fussy sets and knowledge discovery (RSKD'93)", Workshops in Computing, SpringerVerlag and British Coputer Society, London, Berlin,(1994).

[58] W. Ziarko, "Variable precision rough set model", Journal of Computer and System Sciences, Vol.46, (1993), pp.39-59. 